\title{
Determination and Temperature Dependence of $n$-Octanol/Water Partition Coefficients for Seven Sulfonamides from (298.15 to 333.15) K
}

\author{
Zhang Congliang, Wang Yan, and Wang Fuan \\ College of Chemical Engineering, Zhengzhot Lhwersity, Zhengzhou $450002, P$. R. China. ${ }^{*}$ E-mail: zhangcl201azzueducn \\ Received November 22, 2006
}

\begin{abstract}
A shake-flask method was used to determine the $n$-octanol/water partition coefficients of sulfamethazine. sulfadimethoxine, sulfamethoxydiazine. sulfamonomethoxine. sulfamethoxazole, sulfaquinoxaline and sulfachloropyrazine from ( 298.15 to 333.15 ) K. The results showed that the $n$-octanol/water partition coefficient of each sulfonamide decreased with the increase of temperature. Based on the fluid phase equilibrium theory. the thermodynamic relationship of $n$-octanol/water partition coefficient depending on the temperature is proposed. and the changes of enthalpy, entropy, and the Gibbs free energy function for sulfonamides partitioning in $n$ octanol/water are determined. respectively. Sulfonamides molecules partitioning in $n$-octanol/water is mainly an enthalpy driving process. during which the order degrees of șistem increased. The temperature effect coefficient of $n$-octanol/water partition coefficient is discussed. The results show that its magnitude is the same as that of values in the literature.
\end{abstract}

Key Words : Sulfonamide. n-Octanolwater partition coefficient, Tenperature dependence

\section{Introduction}

Sulfonamides also known as a group of șynthetic organic drugs. derived chiefly from sulfanilamide chemically similar to PABA and capable of inhibiting bacterial growth and activity by interfering with the metabolic processes in bacteria that require PABA are extensively used in agriculture to prevent diseases in livestock and treat illness. Subsequently. the potential exists for quantities of these drugs to be excreted as the parent compound and/or metabolites and enter the environment due to the spreading of manure and slurry on agricultural land. or direct deposition by grazing livestock. ${ }^{1-3}$ The fundamental research for their environmental behavior evaluation. biological activity mechanics. interaction mechanics between drug molecules and receptor molecules. and designing new sulfanilamide in the molecular level. is eager to be proceeded. The $n$-octanol/water partition coefficient $\left(P_{\text {ow }}\right)$. which is the quantitative parameter for accessing the interaction between dnig and biofilm, ${ }^{4.5}$ is one of the most important parameters employed for estimating a chemical's environmental fate and toxicity." It is the important factor for determining the drug molecular transfer permeation, absorption distribution and elimination: the key datum for estimating environmental properties such as solubility adsorption coefficient and enrichment factor: and applied widely in forecasting transfer behavior across biofilm for drug molecule. protein incorporation. receptor affinity and biological activity. $P_{0 x}$ defined as the ratio of chemical concentration in the $n$-octanol phase to its concentration in the aqueous phase at equilibrium and expressed as the logarithm of partition coefficient. has been used to predict about acute toxicity and partitioning in the environment. In determining sulfonamides transferring in the environment. and assessing their risk to terrestrial and aquatic ecosystems. it is necessary to know the $n$-octanol/water partition coeffi- cient data of sulfonamides. but only a limited amount of partition coefficient data of sulfonamides have been reported at $298.15 \mathrm{~K}^{7.8}$ In this work. the $n$-octanol/water partition coefficients of sulfonamides have been measured by a shake-flask method from 298.15 to $333.15 \mathrm{~K}$. Based on the fluid phase equilibrium theory the thermodynamic relationship of $n$-octanol/water partition coefficient changing with temperature is proposed. and the entropy change. the enthalpy change, and the Gibbs free energy function change for sulfonamides partitioning in $n$-octanol/water are determined. respectively. The regularity of influence temperature on the $n$-octanol/water partition coefficient is discussed. ${ }^{9}$

\section{Experimental Section}

Materials. Sulfonamides: sulfamethazine. sulfadimethoxine, sulfamethoxydiazine, sulfamonomethoxine, sulfamethoxazole. sulfaquinoxaline and sulfachloropyrazine. obtained from Daming Biotech. Co. Ltd. was further purified by recrystallization from aqueous solutions. After filtration and drying. their purity were determined by UV spectrometry (type UV-2401PC. Shimadzu Co. Ltd). to be 0.996 in mass fraction. Water used in experiments was double distilled water.

Apparatus and procedure. The $n$-octanol/water partition coefficient was measured by a shake-flask method. ${ }^{10}$ Both solvents were mutually saturated before performing the experiments. Solutions of well known concentration, about 5 $\times 10^{-5}$ mole $\mathrm{L}^{-1}$ of sulfonamides. were prepared in aqueous buffer solutions. Then $10.0 \mathrm{~mL}$ of $n$-octanol were added to $10.0 \mathrm{~mL}$ of the aqueous sulfonamide solution in glass flasks. The mixtures were then stirred in a mechanical shaker for 1 h. Samples were allowed to stand in water baths (type 501 . Shanghai Laboratory Instrument Works Co. Ltd.) kept at the appropriate temperature $( \pm 0.02 \mathrm{~K})$ for at least $72 \mathrm{~h}$. After 
Table 1. The comparison of values of measurement and reterences for $n$-octanol/water partition coefticients $\left(\log P_{\text {ow }}\right)$ of some sulfonamides at $298.15 \mathrm{~K}$

\begin{tabular}{lccc}
\hline sultonamide & $\log P_{\text {owexp }}$ & $\log P_{\text {owief }}$ & rel dev $/ \%$ \\
\hline sulfamethazine & 0.2598 & 0.257911 & 0.73 \\
sultamethoxazole & 0.8869 & 0.900012 & -1.5 \\
\hline
\end{tabular}

this time the aqueous phases were isolated and the concentrations were determined by measuring the UV absorbance. The partition coefficients were calculated by mass balance. All the partitioning experiments were repeated at least three times.

The $n$-octanol/water partition coefficient of sulfamethazine and sulfamethoxazole listed in Table $I$ is measured. respectively. to complete the data reported in literature. ${ }^{11.12}$

The $n$-octanol/water partition coefficient is defined as

$$
P_{\text {ow }}=\frac{c_{0}}{c_{\text {r }}}
$$

where $P_{\text {ow }}$ is the $n$-octanol/water partition coefficient of sulfonamide. $\mathcal{c}_{v}$ is the concentration of the sulfonamide in the $n$-octanol phase at equilibrium, $c_{n}$ is the concentration of the sulfonanide in the aqueous phase at equilibrium. $P_{v w}$ is actually the phase equilibrium constant for sulfonanide partitioned in the $n$-octanol phase and the aqueous phase saturated with each other at some temperature.

The result showed that the deviations of the measured $n$ octanol/water partition coefficient from the literature values $^{11,12}$ are less than $2 \%$. In this work. the uncertainty of the logarithm for experimental $n$-octanol/water partition coefficient was estimated to be less than 0.0005 .

\section{Results and Discussion}

The measured $n$-octanol/water partition coefficients $\left(\log P_{\mathrm{ow}}\right)$ of sulfamethazine. sulfadimethoxine. sulfamethoxydiazine. sulfamonomethoxine. sulfamethoxazole. sulfaquinoxaline

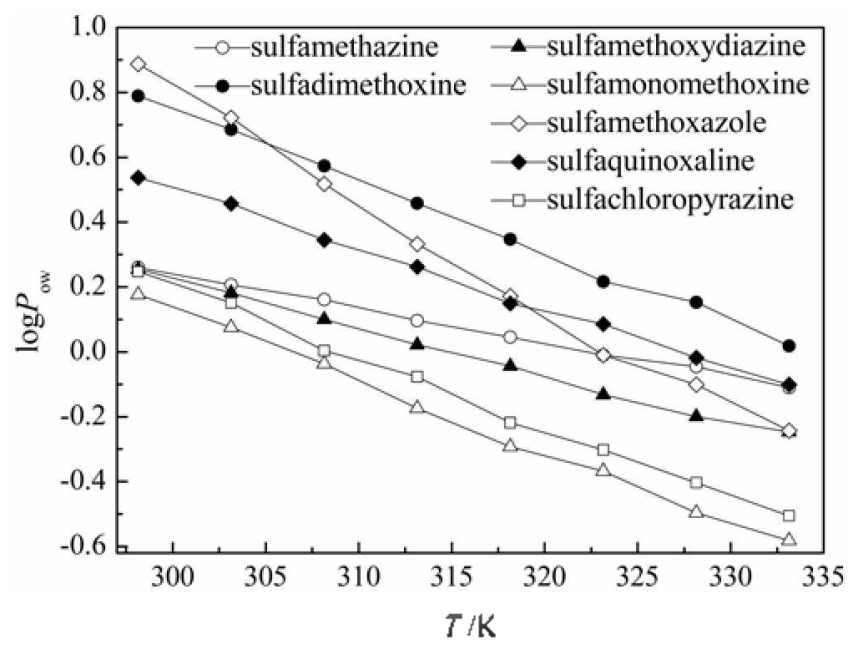

Figure 1. $n$-Octanol/water partition coefficients $\left(\log P_{\text {ow }}\right)$ of seven sulfonamides at different temperatures. and sulfachloropyrazine at different temperatures $T$ are shown in Figure 1. From Figure 1. it can be found that logarithm of the $n$-octanol/water partition coefficient is inversely proportional to $T$.

Thermodynamic theory for simulating biofilm partitioning behavior: Hydrophobic parameter is defined as the ratio of chemical concentration in the lipid phase to its concentration in the aqueous phase at equilibrium. and hydrocarbon/water used as its best solvent. Due to most drugs slightly soluble in hydrocarbon. n-octanol/water is usually used as the ideal solvent for simulating biofilm.

The Gibbs free energy function change $\left(\Delta G_{k \rightarrow 1}^{\circ}\right)$ of interaction between drug and biofilm can be calculated according to the enthalpy change $\left(\Delta H_{x^{\prime} \rightarrow 1}^{o}\right)$ and the entropy change $\left(\Delta S_{u \rightarrow l}\right)$ in the partitioning process.

$$
\Delta G_{\mathrm{u}^{\prime} \rightarrow l}^{\circ}=\Delta H_{\mathrm{u}^{\prime} \rightarrow 1}^{\circ}-T \Delta S_{\mathrm{w}^{\prime} \rightarrow 1}^{\circ}
$$

where $\Delta G_{n \rightarrow l}^{\circ}$ is the synthesis expression of various interactions in the partitioning process of drug transferring from water phase to lipid phase, can be used in judging the spontaneity of partitioning process, assignment degree of drug in two phase, and relation to $n$-octanol/water partition coefficients $\left(P_{\mathrm{ow}}\right)$.

$$
\Delta G_{\mathrm{u}^{\prime} \rightarrow l}^{\circ}=-2.3026 R T \log P_{\mathrm{ow}}
$$

where $R$ and $T$ stand for the universal gas constant and the absolute temperature, respectively. $P_{\mathrm{cw}}$ is the partition equilibrium constant for sulfonamides in $n$-octanol/water system Equation 3 states the thermodynamic relationship between the partition equilibrium constant and the Gibbs free energy function change in the partitioning process.

With the introduction of Equation 3 into Equation 2. logarithm of the $n$-octanol/water partition coefficient can be obtained.

$$
\log P_{\mathrm{ow}}=-\frac{\Delta S_{w \rightarrow 1}^{o}}{2.3026 R T}+\frac{\Delta H_{\mathrm{n} \rightarrow l}^{o}}{2.3026 R}
$$

$\Delta H_{n^{\prime} \rightarrow l}^{o}$ and $\Delta S_{n^{\prime} \rightarrow l}^{\infty}$ are all definite value under certain temperature, so $\log P_{\mathrm{cw}}$ is linear relation to $T^{-1} . \Delta H_{\mathrm{r} \rightarrow l}^{\circ}$ and $\Delta S_{k \rightarrow l}^{\infty}$. calculated by intercept and slope coefficient of the direct line. are enthalpy change and entropy change in the process of drug transferring from water phase to lipid phase. respectively. By studying the thermodynamics for partitioning process of simulating biofilm. and the contribution to $\Delta G_{n \rightarrow l}^{o}$ on $\Delta H_{k \rightarrow l}^{c}$ and $\Delta S_{n \rightarrow l}^{\infty}$ in the partitioning process. the interaction mechanism between drug and simulating biofilm can be predicted.

Partition thermodynamic behavior for $n$-octanol/water system. Owing to their molecules containing basic groups such as $-\mathrm{NH}_{2}$. acidic groups such as $>\mathrm{SO}_{2}$, and groups with different characteristics such as $-\mathrm{CH}_{3} .-\mathrm{OCH}_{3} . \mathrm{Cl}$, sulfonamides perhaps involve various forces such as electrostatic force. hydrogen bond. hydrophobic interaction, and stereoscopic effect in $n$-octanol/water partitioning process. ${ }^{1+4}$

Using least square method, $\Delta G_{n^{\prime} \rightarrow l}^{0}, \Delta H_{n^{\prime} \rightarrow l}^{o} . \Delta S_{n^{\prime} \rightarrow l}^{\infty}$ and related correlation coefficient for every system listed in Table 2. is calculated by the experimental data presented in 
Table 2. The value of $\Delta H_{u^{\prime} \rightarrow l}^{o}$ and $\Delta S_{H^{\prime} \rightarrow l}^{\prime}$ function at $308.15 \mathrm{~K}$

\begin{tabular}{|c|c|c|c|c|}
\hline sultonamide & $\begin{array}{l}\Delta H_{w \rightarrow l}^{o} \\
/ \mathrm{k}_{\mathrm{T}}^{\mathrm{T}} \mathrm{mol}^{-1}\end{array}$ & $\begin{array}{c}\Delta S_{\mathrm{u}}^{\circ} \rightarrow I \\
\mathrm{~J}_{\mathrm{m}} \mathrm{I}^{-1} \mathrm{~K}^{-1}\end{array}$ & $\begin{array}{l}\Delta G_{\mathrm{w} \rightarrow I}^{O} \rightarrow I \\
/ \mathrm{k}^{\mathrm{I}} \mathrm{mlol}^{-1}\end{array}$ & $\begin{array}{l}\text { comelation } \\
\text { coetficient }\end{array}$ \\
\hline sulfamethazine & -20.00 & -62.00 & -0.8947 & 0.9988 \\
\hline sulfadinethosine & -41.83 & -124.9 & -3.342 & 0.9988 \\
\hline sultamethoxydiazine & -28.00 & -89.00 & -0.5747 & 0.9991 \\
\hline sultannonomethosine & -42.03 & -137.4 & -0.3098 & 0.9988 \\
\hline sultamethoxazole & -62.45 & -192.7 & -3.069 & 0.9989 \\
\hline sultaquinoxaline & -34.98 & -106.9 & -2.039 & 0.9991 \\
\hline sulfaclloropyrazine & -41.25 & -133.5 & -0.1120 & 0.9989 \\
\hline
\end{tabular}

Figure 1 through Equation 2 and Equation 4. From Table 2. it can be found that $\Delta H_{w \rightarrow \text { i }}^{o}$ for seven sulfonamides partitioning in $n$-octanol/water system are all negative, which shows that the partitioning process is exothernic. It is perhaps due to interactions between drug molecules and $n$ octanol molecular films being more powerful than those between drug molecules and water molecules, so that energies supplied by forming new bonds exceed those needed by breaking old bonds. which shows the system releasing heat outside. In another word, $n$-octanol molecules solvate drug molecules more easily than water molecules. ${ }^{\text {ls }}$ The powerful interactions between drug molecules and $n$ octanol molecular films are the synergistic consequences of electrostatic force, hydrogen bond, hydrophobic interaction. and so on. $\Delta S_{w \rightarrow I}^{\infty}$ for seven sulfonamides partitioning in $n$ octanol/water system are all negative, which shows that the turbidity of system in the partitioning process doesn't increase. otherwise the system gets ordered. It is indicated that conformations of sulfonamides molecules adapt furthermore to $n$-octanol molecular layers.

In a word, sulfonamides molecules partitioning in $n$ octanol/water is mainly an enthalpy driving process, releasing heat. $n$-octanol solvating drug molecules more easily than water. The entropy changes for sulfonamides molecules partitioning in $n$-octanol/water are all negative. and the order degrees of systems increase.

Temperature dependence of $n$-octanol/water partition coefficients for sulfonamides. The temperature effect coefficient of $n$-octanol/water partition coefficient is defined as the ratio of the change logarithm of $n$-octanol/water partition coefficient to the change of the temperature and expressed as $\mathrm{d}\left(\log P_{\mathrm{OW}}\right) / \mathrm{dT}$. From Equation 4 . the temperature effect coefficient of $n$-octanol/water partition coefficient shown in Figure 2 can be calculated according to

$$
\frac{d\left(\log P_{o n}\right)}{d T}=\frac{\Delta H_{\mathrm{r} \rightarrow \rightarrow l}^{\circ}}{2.3026 R T^{2}}
$$

From Figure 2, it can be found that the temperature effect coefficients of $n$-octanol/water partition coefficient for sulfonamides in this study are all small. negative and similar to the literature values ${ }^{16}$ in the order of magnitude. However. from the viewpoint of the fluid phase equilibrium theory, the effect on $n$-octanol/water partition coefficient cannot be ignored when temperature varies drastically. It is necessary

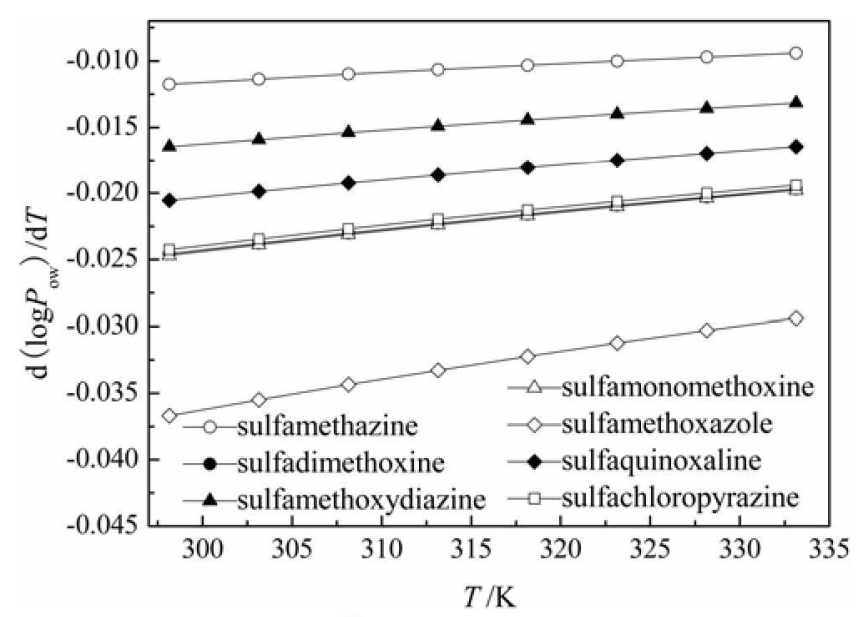

Figure 2. Temperature effect coefficients of $n$-octanol/water partition coefficients $\left(\log P_{\text {oni }}\right)$ for seven sulfonamides.

that the temperature should be controlled exactly on determining the $n$-octanol/water partition coefficient.

According to Scatchard-Hildebrand's theory, ${ }^{17}$ the solubility of solute in the solvent is the largest when the solubility parameter of solute and the solvent are same. The values of the solubility parameter of sulfamethazine $\left(\delta=26.8 \mathrm{MPa}^{1 / 2}\right)$, sulfadimethoxine ( $\delta=26.9 \mathrm{MPa}^{\mathrm{l}}{ }^{2}$ ), sulfamethoxydiazine ( $\delta$ $\left.=27.3 \mathrm{MPa}^{1: 2}\right)$. sulfamonomethoxine $\left(\delta=27.3 \mathrm{MPa}^{1 / 2}\right)$, sulfamethoxazole $\left(\delta=27.2 \mathrm{MPa}^{1: 2}\right)$. sulfaquinoxaline $(\delta=$ $\left.27.9 \mathrm{MPa}^{1: 2}\right)$ and sulfachloropyrazine $\left(\delta=27.9 \mathrm{MPa}^{1 / 2}\right)$, were calculated by the Fedors' group contribution method 17. Solubility parameter for water $\left(\delta=47.9 \mathrm{MPa}^{1 / 2}\right)$ and $n$ octanol ( $\delta=20.0 \mathrm{MPa}^{1 / 2}$ ). come from CRC handbook. ${ }^{17}$ The values of the solubility parameter for sulfamethazine and sulfamethoxazole. respectively. show perfect consistency with the literature values. ${ }^{18.19}$ From the values of solubility parameters, it can be predicated that the difference of the solubility parameter of every sulfonamide and water is larger than that of each sulfonamide and n-octanol. so the logarithm of $n$-octanol/water partition coefficient of each sulfonamide at $298.15 \mathrm{~K}$ is positive.

\section{Conclusion}

A shake-flask method was used to determine the $n$ octanol/water partition coefficients of sulfamethazine. sulfadimethoxine. sulfamethoxydiazine. sulfamonomethoxine. sulfamethoxazole. sulfaquinoxaline and sulfachloropyrazine from $(298.15$ to 333.15$) \mathrm{K}$. The results showed that the $n$ octanol/water partition coefficient of each sulfonamide decreased with the increase of temperature. Based on the fluid phase equilibrium theory. the thermodynamic relationship of n-octanol/water partition coefficient depending on the temperature is proposed and the changes of enthalpy. entropy, and the Gibbs free energy function for sulfonamides partitioning in $n$-octanol/water are determined. respectively: Sulfonamides molecules partitioning in $n$-octanol/water is mainly an enthalpy driving process. during which the order degrees of system increased. The temperature effect coeffi- 
cient of $n$-octanol/water partition coefficient is discussed. The results show that its magnitude is the same as the values in the literature.

Acknowledgment. This research was financed by the Henan Province Natural Science Fund of P. R. China for basic research under Grant No. 0611033400.

\section{References}

1. Kay. P.: Blackwell. P. A.: Boxall. A. B. A. Emironmental Pollation 2005. 134.333.341.

2. Sarmah. A. K.: Meyer. M. T.: Boxall. A. B. A. Chemosphere 2006, 65, 725-759.

3. El-Gaby. M. S. A.: El-Sharief. A. M. Sh.: Atalla A. A.: El-Adas: A. A. A. M. J. Chin. Chem Soc. 2004.51, 327-333.

4. Ong. S.: Liu. H.: Pidgeon. C. J. Chrontatogr: 1996. 728. 113-128.

5. Sun. J.: Wang. S. J.: Chen. G. Acta Pharm. Sin. (China) 2003. 38. $475-480$.

6. Asami, S.; Saitoh. K. Chemistry Letters 2006, 35. 196-197.

7. Martinez, F.: Gomez. A. Journal of Solution Chemishy 2001, 30. 909-923.
8. Martinez. F.: Avila. C. M.: Gomez. A. J. Braz. Chent. Soc. 2003. 1. $803-808$.

9. Bahadur. N. P.: Shiu. W.: Mackay. D. J. Chent Eng. Data 1997. 42. $685-688$.

10. Wang, F. A.; Jiang. Y. L. Moleculor Thenmodnamics and Chromatographic Retention; China Meteorology Press: Beijing. 2001: pp 40-65.

11. Martinez. F.: Gomez. A. Phos Chent Liq 2002. +0.411-420.

12. Jain. N.: Yalkowsky. S. H. J. Pharn. Sei. 2001. 90.234-252.

13. Ottiger. C: Wunderli-Alenspach. H. Pham. Res. 1999. 16, 643650

14. Prausnitz, J. M.; Lichtenthaler. R. N.; Azevedo. E. G. Molecular Thernodnamics of Fluid-Phase Equilibria. 3rd ed.: Prentice Hall: Upper Saddle River. N.T. 1999: pp 645-647.

15. Wang. L. C.: Wang. F. A. Fluid Phase Equilibria 2004. 226. 289293.

16. Sangster. J. J. Phus. Chem. Ref. Data 1989, 18. 1111-1229.

17. Barton, A. F. M. CRC Handhook of Solubility Parameters and Other Cohesion Parameters. 2nd ed: CRC Press: Boca Raton. FL. 1991: pp 99-148.

18. Hancock. B. C.: York. P.: Rowe. R. C. Inter. J. of Pham. 1997. 148. $1-21$.

19. Hanaee. J; Jourban, A ; Dastmalchi. S.: Adiblia, K; Mizazadeh. A.; Barzegarjalali. M. D.tRU 2005. 13, 37-45. 